\title{
Liposome/Graphene Oxide Interaction Studied by Isothermal Titration Calorimetry
}

\author{
Po-Jung Jimmy Huang, Feng Wang and Juewen Liu* \\ Departments of Chemistry, Waterloo Institute for Nanotechnology, Waterloo, Ontario, Canada, N2L 3G1. \\ Supporting Information Placeholder
}

\begin{abstract}
The interaction between graphene oxide (GO) and lipid bilayers is important for fundamental surface science and many applications. In this work, isothermal titration calorimetry (ITC), cryo-TEM, and fluorescence spectroscopy were used to study the adsorption of three types of liposomes. Heat release was observed when GO was mixed with zwitterionic DOPC liposomes, while heat absorption occurred with cationic DOTAP liposomes. For comparison, anionic DOPG liposomes released heat when mixed with DOTAP. DOPC was adsorbed as intact liposomes but DOTAP ruptured and induced stacking and folding of GO sheets. This study suggests the release of more water molecules from the GO surface when mixed with DOTAP liposomes. This can be rationalized by the full rupture of the DOTAP liposomes interacting with the whole GO surface, including hydrophobic regions; while DOPC liposomes only interact with a small area on GO near the edge, which is likely to be more hydrophilic. This interesting biointerfacial observation has enhanced our fundamental understanding of lipid/GO interactions.
\end{abstract}

\section{INTRODUCTION}

Graphene is a single layer of graphite possessing a huge specific surface area. ${ }^{1}$ Many unique electric, thermal, and optical properties of graphene were reported in the past decade. ${ }^{2}$ At the same time, graphene was interfaced with various biomolecules to prepare drug delivery vehicles, biomaterials, and sensor devices. ${ }^{3-10}$ A fundamental aspect is the interaction between graphene and the cell membrane, whose main component is phospholipid. ${ }^{11-18}$ Since pristine graphene is difficult to disperse in water, graphene oxide (GO) rich in hydroxyl, epoxy and carboxyl groups is often used for most biological applications.

To understand the interaction between lipids and graphenebased materials, both supported lipid bilayers and colloidally dispersed liposomes have been used. For example, Frost et al adsorbed cationic liposomes on GO via electrostatic interactions, ${ }^{12}$ liposome fusion onto the GO surface was suggested based on quartz crystal microbalance (QCM) and atomic force microscopy (AFM). Loh and co-workers interfaced liposomes of various charges with pristine graphene sheets deposited on a wafer, where they reported liposome fusion. ${ }^{11}$ Molecular dynamics simulation showed the insertion of a graphene sheet between the hydrophobic tails of a lipid bilayer, which is equivalent to supported monolayers. ${ }^{13}$ We reported adsorption of zwitterionic phosphocholine (PC) liposomes by GO of different oxidation levels. ${ }^{19}$ PC liposomes retained their integrity on GO; leakage occurred on reduced GO (rGO); while full liposome rupture took place on pristine graphene. Surface enhance IR spectroscopy indicated the contribution of electrostatic, hydrophobic, and hydrogen bonding interactions. ${ }^{20} \mathrm{We}$ proposed that hydrogen bonding is the major contribu- tor for PC liposome adsorption based on urea, salt, and $\mathrm{pH}$ dependent studies. ${ }^{19,21}$

Despite these progresses, some important questions remain to be answered such as the thermodynamic nature of the adsorption reaction and the role of surface water. Isothermal titration calorimetry (ITC) is a biophysical technique that measures the heat released or absorbed during a binding reaction, from which rich thermodynamic information can be obtained. ITC has been widely used for studying ligand binding to proteins and nucleic acids. ITC is also useful for studying molecular adsorption by inorganic and polymeric surfaces. For example, adsorption of nucleotides, nucleic acids, and proteins by GO was reported. ${ }^{22-24}$ In this work, we employed ITC to study the adsorption between liposomes and GO.

\section{MATERIALS AND METHODS}

Chemicals. All the phospholipid samples were from Avanti Polar Lipids (Alabaster, AL). GO was purchased from ACS Material, LLC (Medford, MA). 4-(2-hydroxyethyl)-1piperazineethanesulfonic acid (HEPES), 2-(Nmorpholino)ethanesulfonic acid (MES), and $\mathrm{NaCl}$ were from Mandel Scientific (Guelph, ON, Canada). Milli-Q water was used to prepare all the buffers and solutions.

Preparation of liposomes. Liposomes were prepared using the standard extrusion with a mini-extruder from Avanti Polar Lipids. DOPC, DOPG, or DOTAP $(2.5 \mathrm{mg})$ was respectively dissolved in chloroform. Rh-labeled liposomes were prepared by including $1 \%$ (w/w) Rh-PE (2-dioleoyl-sn-glycero-3-phosphoethanolamine-N(lissaminerhodamine B sulfonyl) (ammonium salt). Chloroform was then removed under a gentle $\mathrm{N}_{2}$ flow in a fume hood and trace amounts of residual chloroform was removed by storing the samples in a vacuum oven overnight at room temperature. The dried lipid films were kept under a $\mathrm{N}_{2}$ environment and then stored at $-20{ }^{\circ} \mathrm{C}$ prior to use. To prepare liposomes, the dried lipid films were hydrated with $0.5 \mathrm{~mL}$ buffer A $(100 \mathrm{mM} \mathrm{NaCl}, 10 \mathrm{mM}$ HEPES, $\mathrm{pH}$ 7.6) at room temperature with occasional sonication for at least $2 \mathrm{~h}$ (lipid concentration $=5 \mathrm{mg} / \mathrm{mL}$ ). The resulting cloudy suspension was extruded through two stacked polycarbonate membrane (pore size $=100 \mathrm{~nm}$ ) for 21 times. After extrusion, the lipid solution appeared to be transparent, indicating formation of liposomes.

Size and $\zeta$-potential measurement. The size and $\zeta$-potential of our liposomes $(50 \mu \mathrm{g} / \mathrm{mL})$ was characterized using dynamic light scattering (DLS) in $10 \mathrm{mM}$ HEPES buffer, $\mathrm{pH} 7.6$ at $25^{\circ} \mathrm{C}$ on a Zetasizer Nano ZS90 (Malvern) with a He-Ne laser $(633 \mathrm{~nm})$ at $90^{\circ}$ collecting angle. The data were analyzed by Malvern Dispersion Technology Software 4.20.

ITC titration. All the ITC experiments were performed with a MicroCal 200 instrument. All the reagents were dissolved in buff- 
er B (10 mM MES, pH 6.0) and degassed prior to the titration. For most experiments, a total of $42.5 \mu \mathrm{L}$ liposomes $(5 \mathrm{mg} / \mathrm{mL})$ were injected $(0.5 \mu \mathrm{L}$ for the first injection, and $6 \mu \mathrm{L}$ each for the subsequent 7 injections) into the reaction cell containing $250 \mu \mathrm{L}$ of GO $(240 \mu \mathrm{g} / \mathrm{mL})$ at $25^{\circ} \mathrm{C}$. The titrant was injected at 5 min intervals to ensure that the titration peak returned to the baseline before the next injection. For the DOTAP sample, due to the high background heat, we titrated GO into DOTAP. In this case, 1 $\mathrm{mg} / \mathrm{mL}$ of GO was titrated into $2 \mathrm{mg} / \mathrm{mL}$ of DOTAP. For a control experiment, $5 \mathrm{mg} / \mathrm{mL}$ of DOPG was titrated into $2 \mathrm{mg} / \mathrm{mL}$ of DOTAP. A background titration, consisting of an identical titrant solution but with the buffer solution in the reaction cell only, was recorded each time to account for the heat of dilution and background signal.

Cryo-TEM. GO $(40 \mu \mathrm{L}, 200 \mu \mathrm{g} / \mathrm{mL})$ was mixed with $80 \mu \mathrm{L}$ of $2.5 \mathrm{mg} / \mathrm{mL}$ DOPC or DOTAP liposomes in buffer B at room temperature overnight. The excess amount of liposome was removed after centrifugation. TEM samples were prepared by spotting $5 \mu \mathrm{L}$ of the liposome suspension on a carbon-coated copper TEM grid (treated with plasma to ensure surface was hydrophilic) in a humidity controlled chamber (FEI Vitrobot). The humidity was set to be 95 to $100 \%$ during this operation. The grid was blotted with two filter papers for $1.5 \mathrm{sec}$ and quickly plunged into liquid ethane. The sample was then loaded to a liquid $\mathrm{N}_{2}$ cooled cold stage and loaded into a $200 \mathrm{kV}$ field emission TEM (FEI Tecnai G2 F20). The samples were imaged when the temperature was stabilized at $-178^{\circ} \mathrm{C}$.

GO/liposome interaction by visual inspection. The as-received micrometer sized GO $(200 \mu \mathrm{L}, 200 \mu \mathrm{g} / \mathrm{mL})$ was respectively mixed with the three types of liposomes $(10 \mu \mathrm{L}, 5 \mathrm{mg} / \mathrm{mL})$ in Milli-Q water for $5 \mathrm{~min}$. After centrifugation at $6,000 \mathrm{rpm}$ for 10 $\mathrm{sec}$ on a mini-centrifuge, the samples was photographed using a digital camera (Canon PowerShot SD 1200 IS).

Liposome adsorption capacity. To measure the adsorption capacity, $1 \%$ Rh-labeled liposomes were used. In a typical experiment, $5 \mu \mathrm{L}$ DOPC ( $5 \mathrm{mg} / \mathrm{mL})$ was mixed with $100 \mu \mathrm{L}$ of GO (20 $\mu \mathrm{g} / \mathrm{mL}$ ) in $10 \mathrm{mM}$ HEPES ( $\mathrm{pH}$ 7.6). The mixture was incubated for $10 \mathrm{~min}$ and then centrifuged at 15,000 rpm for $15 \mathrm{~min}$. At this speed, the free liposomes were not precipitated, while the DOPC/GO complexes were. By comparing the fluorescence intensity remained in the supernatant $\left(F_{\mathrm{s}}\right)$ with that of the free $\mathrm{Rh}-$ DOPC liposome $\left(F_{0}\right)$, the adsorption capacity of DOPC on each mg of nano-GO was calculated to be $\left(F_{0}-F_{\mathrm{s}}\right) / F_{0}$.

\section{RESULTS AND DISCUSSION}

Adsorption of DOPC liposomes. In this work, three types of liposomes were studied (Figure 1A): DOPC (1,2-dioleoyl-snglycero-3-phosphocholine); DOPG (1,2-dioleoyl-sn-glycero-3phospho-(1'-rac-glycerol)); and DOTAP (1,2-dioleoyl-3-trimethyl ammonium-propane). The corresponding liposomes were prepared by the extrusion method using a polycarbonate membrane with $100 \mathrm{~nm}$ pores. The size of the liposomes were around 110 $\mathrm{nm}$ from dynamic light scattering (Figure S1, Supporting Information). A cryo-TEM micrograph of the DOPC liposomes is shown in Figure 1E. To simplify data interpretation, each liposome was prepared with $100 \%$ of the designated lipid, and their surface charge was confirmed by $\zeta$-potential measurement (Figure 1B). The zwitterionic DOPC has an overall neutral charge, while DOTAP is cationic and DOPG is anionic, all in agreement with their chemical structures.

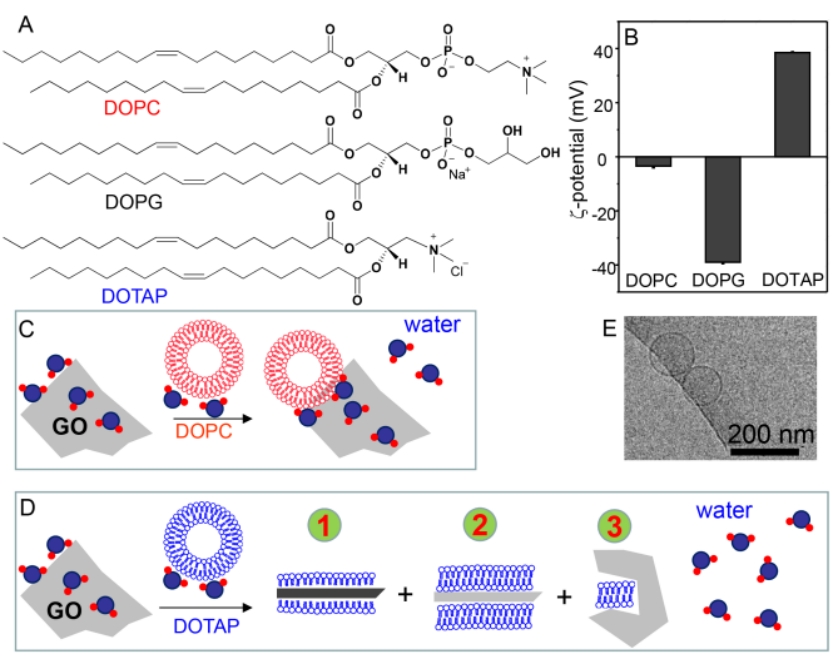

Figure 1. (A) The structures of the three lipids studied in this work, each with a different charge. (B) $\zeta$-potential of the three liposomes (in $10 \mathrm{mM}$ HEPES, pH 7.6). Schematics of GO interacting with (C) zwitterionic DOPC liposomes and (D) cationic DOTAP liposomes. DOPC adsorbs as intact liposomes on the edge of GO. More water molecules are released from GO with the DOTAP interaction and a few possible products are drawn based on our cryo-TEM data. These products may further deform and aggregate. (E) A cryo-TEM micrograph of the free DOPC liposomes.

Our GO sample has an oxygen content of $40.8 \%{ }^{19}$ With its surface carboxyl groups, GO is negatively charged. We respectively mixed each liposome with GO in water. After a brief centrifugation, the DOPC and DOTAP samples precipitated, while the DOPG sample remained stable (Figure 2A). This indicates the lack of GO adsorption by DOPG. Next, to quantitatively measure adsorption capacity, we labeled each liposome with $1 \% \mathrm{Rh}-\mathrm{PE}$ lipid and incubated excess amount of the liposomes with GO. The adsorbed liposomes were calculated from the supernatant fluorescence (Figure 2B). DOPC and DOTAP had a similarly high capacity, while DOPG adsorbed much less, consistent with the results in Figure 2A. It is interesting to note that cationic DOTAP did not adsorb more than neutral DOPC, despite the former has a strong electrostatic interaction with GO.

A
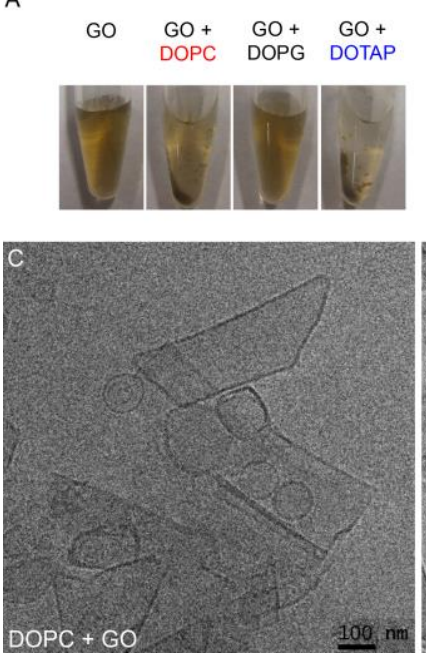
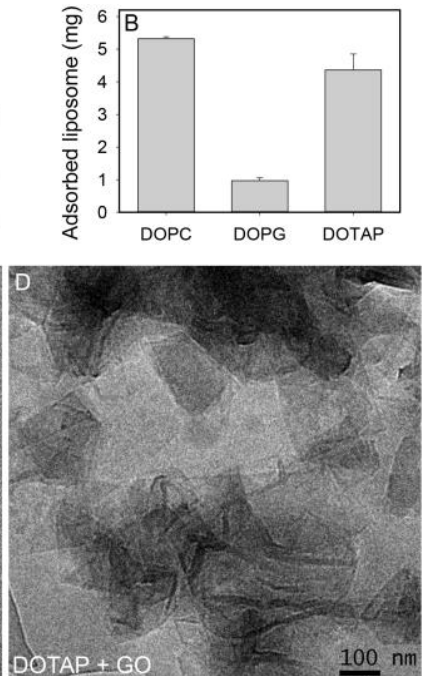
Figure 2. (A) Photographs of the three liposomes mixed with GO in water. (B) Quantification of the adsorption capacity of each mg of GO for each liposome. Cryo-TEM micrographs of GO mixed with (C) DOPC and (D) DOTAP liposomes. In (C) intact liposomes are observed on the edge of the GO sheets, while in (D) the GO sheets are folded and aggregated with no liposome observed.

For the following studies, we focus on the DOPC and DOTAP systems since they are spontaneously adsorbed by GO. DOTAP adsorption is believed to take place through electronic attraction, while DOPC adsorption might involve hydrogen bonding. ${ }^{19,21}$ To gain a better thermodynamic understanding, the adsorption reactions were followed by ITC. In a typical experiment, small volumes of liposomes were injected into a large volume of GO and the rate of heat released or absorbed was recorded as a function of time.

First, the zwitterionic DOPC liposomes were tested. Each time, $6 \mu \mathrm{L}$ of DOPC ( $38.4 \mathrm{nmol}$ or $30 \mu \mathrm{g}$ lipid) was injected into the GO sample $(60 \mu \mathrm{g})$. The experiment was initially performed in a salt-free buffer containing just $10 \mathrm{mM}$ MES (pH 6.0). A relatively large amount of heat was released during the first two injections (Figure 3A), after which the heat started to decrease. The heat at the 6th injection (total $180 \mu \mathrm{g}$ lipid injected) was very small, suggesting surface saturation. From the adsorption capacity in Figure 2B, $60 \mu \mathrm{g}$ GO can maximally adsorb $\sim 300 \mu \mathrm{g}$ DOPC liposomes. Since the GO surface is heterogeneous with both highly oxidized regions and carbon-rich regions, ${ }^{25,}{ }^{26}$ we reason that DOPC liposomes are first adsorbed on the higher affinity sites releasing more heat followed by the lower affinity sites with less heat. For most injections, the peaks were quite sharp, but the background was not fully recovered, which might be due to a slow reorganization of the system to a more stable state. We also measured the adsorption kinetics using Rh-labeled DOPC liposomes based on fluorescence quenching (Figure 3D). At all the tested salt concentrations, an initial fast adsorption phase was observed followed by a slower phase, consistent with the ITC titration results. Note that precipitation of the DOPC/GO complex may also contribute to the gradual fluorescence dropping, and thus we do not quantitatively discuss the fluorescence-based kinetic data here.

Based on the heat released from the first peak, we calculated the enthalpy of this reaction to be just $-0.35 \mathrm{kcal} / \mathrm{mol}$ of DOPC lipid assuming each lipid molecule was adsorbed by GO. However, it needs to be pointed out that DOPC liposomes are adsorbed without fusion onto the GO surface. ${ }^{19,}{ }^{27} \mathrm{We}$ also observed from cryo-TEM that DOPC liposomes tend to attach to the edge of the GO sheets (Figure 2C). ${ }^{19}$ Therefore, the number of DOPC lipids in direct contact with the GO surface is only a small fraction of the total lipid, and the $\Delta H=-0.35 \mathrm{kcal} / \mathrm{mol}$ based on the adsorption of all the lipids is not an accurate description of the system. For example, if only 5\% (arbitrarily estimated) of lipids were responsible for adsorption, the heat should be $-7 \mathrm{kcal} / \mathrm{mol}$. The small amount of heat and the large noise from ITC precluded us from extracting more quantitative thermodynamic information such as adsorption entropy and free energy. Nevertheless, ITC still tells the exothermic nature of this reaction.
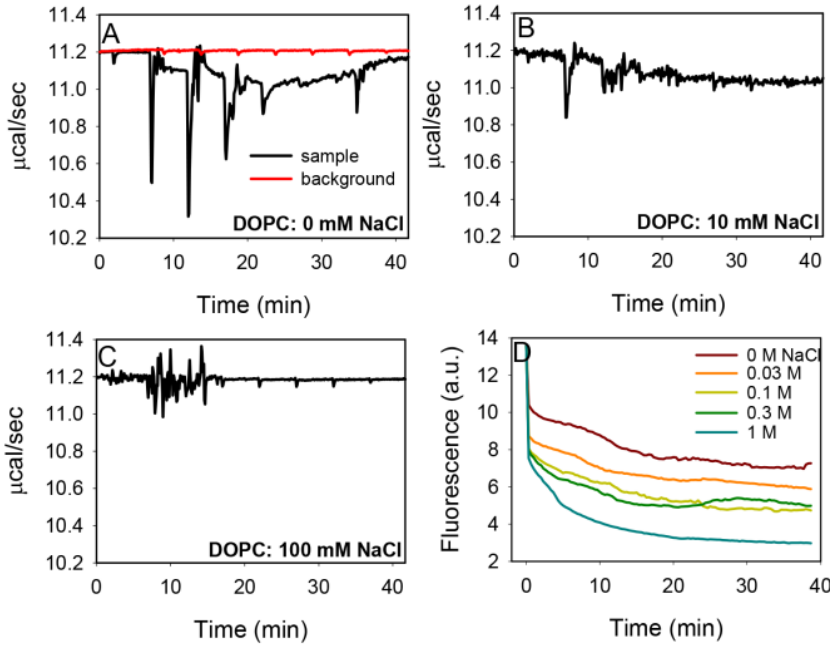

Figure 3. ITC traces of DOPC liposomes titration into GO as with (A) $0 \mathrm{mM}$, (B) $10 \mathrm{mM}$, and (C) $100 \mathrm{mM} \mathrm{NaCl}$. (D) Kinetics of Rh-labeled DOPC adsorption by $\mathrm{GO}$ at different $\mathrm{NaCl}$ concentrations.

It is interesting to notice that the released heat is very sensitive to salt concentration. Even $10 \mathrm{mM} \mathrm{NaCl}$ suppressed the signal (Figure 3B), and $100 \mathrm{mM} \mathrm{NaCl}$ completely abolished heat release (Figure 3C). Our adsorption kinetics data in Figure 3D already indicated that DOPC adsorption by GO takes places at all the salt concentrations, consistent with our previous centrifugation-based measurement. ${ }^{19}$ Actually adsorption appears even faster and more complete with higher salt from the stronger quenching observed.

Since a high salt concentration does not impede adsorption, its lack of heat release indicates the increased entropy contribution of the reaction. We reason that at higher $\mathrm{NaCl}$ concentrations, the negatively charged GO surface (especially near the carboxyl groups) is covere by more counter-ions $\left(\mathrm{Na}^{+}\right)$in the double layer; some might be tightly adsorbed. Most of these ions need to be released to allow DOPC adsorption, which is accompanied with entropy gain but heat absorption, explaining the salt-dependent trend in Figure 3A-C.

Adsorption of DOTAP liposomes. Next we studied adsorption of cationic DOTAP liposomes. We noticed a large amount of background heat when titrating DOTAP liposomes into buffer without GO (Figure 4A, note the difference in the scale of its $y$-axis compared to the other plots), which was attributed to the interaction between the DOTAP liposomes and the inner wall of the ITC chamber. To avoid this artifact, we titrated GO into DOTAP liposomes (instead of DOTAP into GO) for this experiment. By adding DOTAP first into the chamber, we can ensure that all the chamber surface was covered by DOTAP assuming DOTAP can be adsorbed by the wall, and the only interaction we measured was the adsorption between DOTAP and GO. In this case, the background heat of titrating GO into buffer was smaller (red trace, Figure 4B). After subtracting this background, heat absorption instead of release was observed when GO was added to DOTAP (black trace, Figure 4B). A similar amount of heat was absorbed for the first six injections, suggesting quantitative and efficient reactions. The peaks are very sharp and the reaction kinetics are much faster compared to that of the DOPC titration. This is consistent with the strong electrostatic attraction between DOTAP and GO. For the final injection, the heat decreased, indicating the saturation of the DOTAP surface by GO. By comparing Figure 4B with Figure $3 \mathrm{~A}$, it is interesting to note that DOPC adsorption 
released heat while DOTAP absorbed heat (both experiments were carried out in the same buffer without $\mathrm{NaCl}$ ).

In the above titration, each injection added $6 \mu \mathrm{g}$ of GO, which can adsorb $27 \mu \mathrm{g}(0.0387 \mu \mathrm{mol})$ DOTAP liposome (based on the adsorption capacity from Figure $2 \mathrm{~B}$ ). The average heat absorbed is $4.2 \mu \mathrm{cal}$ from the first six injections in Figure $4 \mathrm{~B}$, thus yielding a heat of $0.11 \mathrm{kcal} / \mathrm{mol}$. This is a quite small heat (thermal energy is $0.59 \mathrm{kcal} / \mathrm{mol}$ at $298 \mathrm{~K}$ ) of adsorption. However, considering the whole lipid layer with thousands of lipid molecules interacting collectively with the GO surface (see our cryo-TEM discussion below), the overall adsorption can still be quite strong.

We also noticed that the product from the DOTAP/GO ITC titration to be extensively aggregated. As a technical note here, it is very difficult to clean the ITC chamber after such a titration. To have a better understanding on the interaction between DOTAP liposomes and GO, their mixture was characterized by cryo-TEM (Figure 2D). The GO sheets were indeed extensively aggregated, stacked, and folded. There must be strong attraction forces imposed by the DOTAP liposomes to induce such structures. Unlike DOPC liposome adsorption, where intact liposomes were observed, we cannot find any spherical DOTAP liposomes in the cryo-TEM micrographs, suggesting that the DOTAP liposomes were fully ruptured. By attaching GO to a supported lipid bilayer, Frost et al observed cationic liposomes (with 25\% cationic POEPC lipid and 75\% POPC) fusion onto GO, ${ }^{12}$ and their AFM micrographs after three alternating GO and liposome deposition cycles are quite similar to our cryo-TEM with a simple mixing in solution.

The theoretical surface area of pristine graphene is $2630 \mathrm{~m}^{2} / \mathrm{g}$ (counting both side of the sheet). Our GO sample contained $\sim 40 \%$ oxygen, rendering a maximal possible area of $1.58 \mathrm{~m}^{2} / \mathrm{mg}$ or 0.79 $\mathrm{m}^{2} / \mathrm{mg}$ if we only count one side of the surface. From Figure 2B, each $\mathrm{mg}$ of GO can adsorb $4.5 \mathrm{mg}$ of DOTAP, which has a surface area of $2.4 \mathrm{~m}^{2}$ (if assuming the same headgroup area of 0.6 $\mathrm{nm}^{2}$ as DOPC since we cannot find such data for DOTAP). ${ }^{28}$ Therefore, each GO sheet might take between one and two DOTAP bilayers.

It is known that GO has both highly oxidized domains and more hydrophobic domains rich in carbon. ${ }^{25},{ }^{26}$ Based on the ITC and cryo-TEM data, we propose that the hydrophobic domains on GO are initially adsorbed with ordered water layers. ${ }^{29}$ Releasing these water costs heat (but gains entropy). Unlike DOPC liposome adsorption, where the majority of the GO surface remains unoccupied by lipid (interactions take place mainly on the carboxyl rich hydrophilic edges), DOTAP adsorption results in the spread of the lipid on the whole GO surface. Therefore, a large amount of water molecules released from the GO sheets to form the GO/DOTAP interface, including those originally adsorbed on the hydrophobic regions, which are responsible for heat absorption and entropy gain.

For comparison, we titrated negatively charged DOPG liposomes into DOTAP and a large amount of heat release was observed (Figure 4D). Therefore, the heat absorption when GO was titrated must be related to the surface property of GO beyond its negative charges (both GO and DOPG are negatively charged). The main difference is likely to be the presence of hydrophobic domains in GO, while the DOPG surface is all hydrophilic.
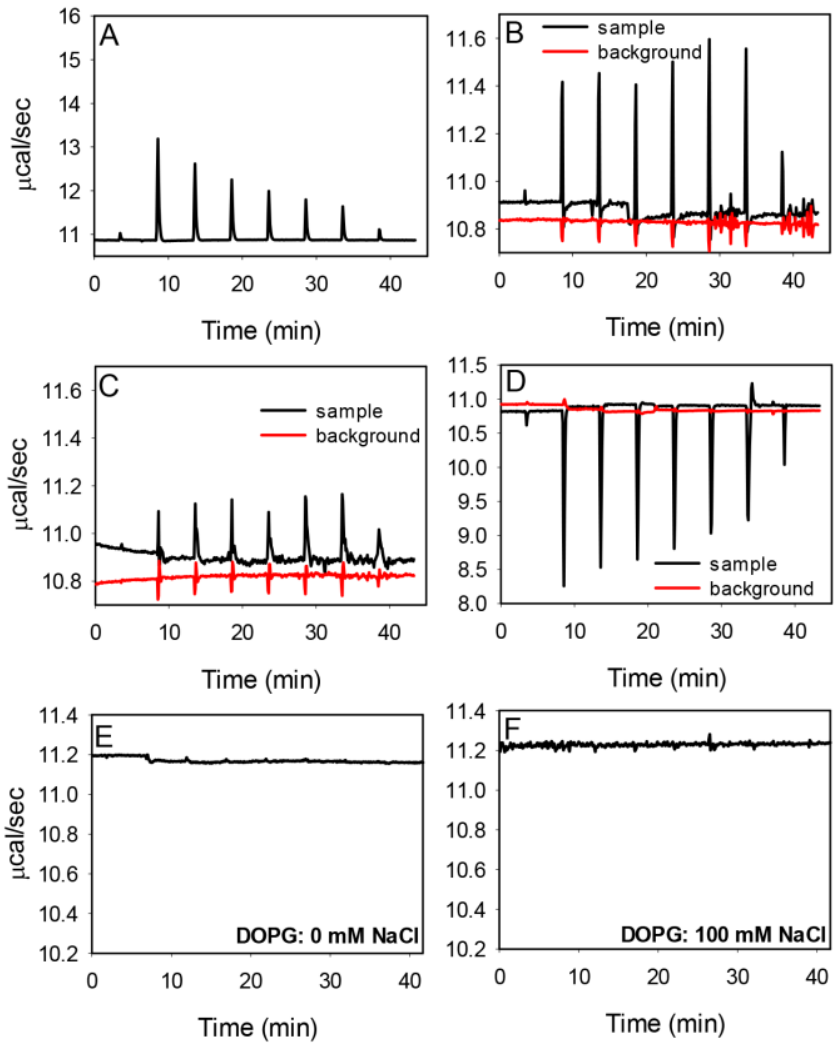

Figure 4. (A) ITC traces of titrating DOTAP liposomes into buffer (10 mM MES, pH 6.0). ITC traces of titrating GO into DOTAP with (B) no $\mathrm{NaCl}$ and (C) $100 \mathrm{mM} \mathrm{NaCl}$. (D) Titrating DOPG liposomes into DOTAP. The upward spikes indicate heat absorption while the downward spikes indicate heat release. Titrating DOPG liposomes into GO (E) with no $\mathrm{NaCl}$ and (F) with $100 \mathrm{mM}$ $\mathrm{NaCl}$.

Based on the above discussion and cryo-TEM, three reaction products between DOTAP and GO were proposed in Figure 1D. It might be that the carbon-rich regions on GO formed product 1 (GO sandwiched between the hydrophobic lipid tails), and highly oxidized regions formed product 2 (supported bilayers). These structures may further stack to produce multilayers. Based on the cryo-TEM, we cannot rule out product 3 either. The heat absorption should be related to the water molecules released during product 1 formation.

Interestingly, while the DOPC liposomes are only sparsely adsorbed on the edge of $\mathrm{GO}, \mathrm{GO}$ has roughly the same adsorption capacity (Figure 2B). Therefore, we can deduce that if the adsorbed DOPC liposomes are full ruptured, they should be able to cover the whole GO surface as well.

The interaction between GO and lipid headgroup was also studied by Leblanc and co-workers using lipid monolayers at the air/water interface. ${ }^{30}$ Electrostatic interactions are proposed to be the main force for GO to interact with both zwitterionic PC lipids and cationic lipids. The electrostatic nature is reflected also in our system by the NaCl-dependent data. Their monolayer system does not however fully mimic lipid bilayers especially consider ruptured liposomes, since the GO sheets are confined in the aqueous phase and cannot touch the hydrophobic tails. ${ }^{13}$

We further measured titrated GO to the DOTAP liposome in the presence of $100 \mathrm{mM} \mathrm{NaCl}$, and the amount of heat absorbed also decreased (Figure 4C). With $100 \mathrm{mM} \mathrm{NaCl}$, the Debye length is $\sim 1 \mathrm{~nm}$, and under this condition, electrostatic interactions are 
still strong enough to allow adsorption and subsequent liposome rupture. Overall, salt decreased the absolute amount of heat (released in the DOPC case or absorbed in the DOTAP case). The difference is likely attributable to the role of water and ions in the double layer. ${ }^{31}$ It is likely that salt screened electrostatic interactions between DOTAP and GO, thus decreasing the number of released water molecules. In the case of DOPC adsorption, water release is not a main factor, and their hydrogen bonding interaction is believed to be mediated by a water molecule. ${ }^{20}$ It is the removal of extra salt ions that consumed the enthalpy.

After studying DOPC and DOTAP liposomes, we further titrated anionic DOPG liposomes to GO. From the aggregation assay (Figure 2A) and fluorescence-based studies (Figure 2B), DOPG does not interact strongly with GO. Indeed, no binding was detected from ITC at either low or high salt concentrations (Figure 4E, F).

Based on these observations, we summarized our findings in Figure 1C, D. Previous studies indicated that water mediates the hydrogen bonding interaction between DOPC liposomes and $\mathrm{GO},{ }^{20}$ and thus the interface between these two is not fully dehydrated. In addition, DOPC and GO interact mainly on the edge of GO, which is more hydrophilic than the carbon-rich domains in the interior. Therefore, only a small number of water molecules are released. On the other hand, more water molecules are released from the DOTAP/GO interaction (interaction covers the whole GO surface), and some of the water molecules are residing on the more hydrophobic regions, accounting for more entropy contributions of this reaction. The interaction with DOTAP is so strong that multi-layered structures and folded GO sheets are possible.

\section{CONCLUSIONS}

In summary, we used ITC, cyro-TEM, and fluorescence spectroscopy to study the interaction between GO and liposomes of different charges. ITC complements previously employed techniques such as AFM and QCM by providing thermodynamic information that can be related to the ions in the electric double layer and adsorbed water on GO. The ITC profiles indicate a large amount of released water molecules for DOTAP liposome interacting with GO. In comparison, DOPC interaction takes place only on a small region of the liposome with fewer water released. The final hybrid materials are also very different based on cryo-TEM studies. These experiments provide valuable information on the biointerface between GO and lipid bilayers.

\section{ASSOCIATED CONTENT}

\section{Supporting Information}

Materials and methods, DLS data. This material is available free of charge via the Internet at http://pubs.acs.org.

\section{AUTHOR INFORMATION}

\section{Corresponding Author}

Email: liujw@uwaterloo.ca

\section{ACKNOWLEDGMENT}

We thank Mr. Robert Harris at the University of Guelph for assistance with cryo-TEM experiments. Funding for this work is from the Natural Sciences and Engineering Research Council of Canada (NSERC).

\section{REFERENCES}

(1) Geim, A. K.; Novoselov, K. S., The Rise of Graphene. Nat. Mater. 2007, 6, 183-191.

(2) Novoselov, K. S.; Falko, V. I.; Colombo, L.; Gellert, P. R.; Schwab, M. G.; Kim, K., A Roadmap for Graphene. Nature 2012, 490, 192-200.

(3) Yang, K.; Feng, L.; Shi, X.; Liu, Z., Nano-Graphene in Biomedicine: Theranostic Applications. Chem. Soc. Rev. 2013, 42, 530-547.

(4) Chen, D.; Feng, H.; Li, J., Graphene Oxide: Preparation, Functionalization, and Electrochemical Applications. Chem. Rev. 2012, 112, 6027-6053.

(5) Wang, Y.; Li, Z. H.; Wang, J.; Li, J. H.; Lin, Y. H., Graphene and Graphene Oxide: Biofunctionalization and Applications in Biotechnology. Trends Biotechnol. 2011, 29, 205-212.

(6) Wang, H.; Yang, R. H.; Yang, L.; Tan, W. H., Nucleic Acid Conjugated Nanomaterials for Enhanced Molecular Recognition. ACS Nano 2009, 3, 2451-2460.

(7) Loh, K. P.; Bao, Q.; Eda, G.; Chhowalla, M., Graphene Oxide as a Chemically Tunable Platform for Optical Applications. Nat Chem 2010, 2, 1015-1024.

(8) Shao, Y. Y.; Wang, J.; Wu, H.; Liu, J.; Aksay, I. A.; Lin, Y. H., Graphene Based Electrochemical Sensors and Biosensors: A Review. Electroanalysis 2010, 22, 1027-1036.

(9) Lu, C. H.; Yang, H. H.; Zhu, C. L.; Chen, X.; Chen, G. N., A Graphene Platform for Sensing Biomolecules. Angew. Chem. Int. Ed. 2009, 48, 4785-4787.

(10) Liu, Z.; Liu, B.; Ding, J.; Liu, J., Fluorescent Sensors Using DNA-Functionalized Graphene Oxide. Anal. Bioanal. Chem. 2014, 406, 6885-6902.

(11) Ang, P. K.; Jaiswal, M.; Lim, C. H. Y. X.; Wang, Y.; Sankaran, J.; Li, A.; Lim, C. T.; Wohland, T.; Barbaros, O.; Loh, K. P., A Bioelectronic Platform Using a GrapheneLipid Bilayer Interface. ACS Nano 2010, 4, 7387-7394.

(12) Frost, R.; Jonsson, G. E.; Chakarov, D.; Svedhem, S.; Kasemo, B., Graphene Oxide and Lipid Membranes: Interactions and Nanocomposite Structures. Nano Lett. 2012, 12, 3356-3362.

(13) Titov, A. V.; Kral, P.; Pearson, R., Sandwiched GrapheneMembrane Superstructures. ACS Nano 2009, 4, 229-234.

(14) Okamoto, Y.; Tsuzuki, K.; Iwasa, S.; Ishikawa, R.; Sandhu, A.; Tero, R., Fabrication of Supported Lipid Bilayer on Graphene Oxide. In Asia-Pacific Interdisciplinary Research Conference 2011, Sandhu, A., Ed. IOP Publishing Ltd: Bristol, 2012; Vol. 352, p 012017.

(15) Tu, Y. S.; Lv, M.; Xiu, P.; Huynh, T.; Zhang, M.; Castelli, M.; Liu, Z. R.; Huang, Q.; Fan, C. H.; Fang, H. P.; Zhou, R. H., Destructive Extraction of Phospholipids from Escherichia Coli Membranes by Graphene Nanosheets. Nat. Nanotechnol. 2013, 8, 594-601.

(16) Li, Y. F.; Yuan, H. Y.; von dem Bussche, A.; Creighton, M.; Hurt, R. H.; Kane, A. B.; Gao, H. J., Graphene Microsheets Enter Cells through Spontaneous Membrane Penetration at Edge Asperities and Corner Sites. Proc. Natl. Acad. Sci. U.S.A. 2013, 110, 12295-12300.

(17) Krishnamoorthy, K.; Veerapandian, M.; Zhang, L.-H.; Yun, K.; Kim, S. J., Antibacterial Efficiency of Graphene Nanosheets against Pathogenic Bacteria Via Lipid Peroxidation. J. Phys. Chem. C 2012, 116, 17280-17287. 
(18) Pham, V. T. H.; Truong, V. K.; Quinn, M. D. J.; Notley, S. M.; Guo, Y.; Baulin, V. A.; Al Kobaisi, M.; Crawford, R. J.; Ivanova, E. P., Graphene Induces Formation of Pores That Kill Spherical and Rod-Shaped Bacteria. ACS Nano 2015, 9, 8458-8467.

(19) Ip, A. C. F.; Liu, B.; Huang, P.-J. J.; Liu, J., Oxidation Level-Dependent Zwitterionic Liposome Adsorption and Rupture by Graphene-Based Materials and Light-Induced Content Release. Small 2013, 9, 1030-1035.

(20) Wu, L.; Zeng, L.; Jiang, X., Revealing the Nature of Interaction between Graphene Oxide and Lipid Membrane by Surface-Enhanced Infrared Absorption Spectroscopy. J. Am. Chem. Soc. 2015, 137, 10052-10055.

(21) Wang, F.; Liu, J., Nanodiamond Decorated Liposomes as Highly Biocompatible Delivery Vehicles and a Comparison with Carbon Nanotubes and Graphene Oxide. Nanoscale 2013, 5, 12375-12382.

(22) Varghese, N.; Mogera, U.; Govindaraj, A.; Das, A.; Maiti, P. K.; Sood, A. K.; Rao, C. N. R., Binding of DNA Nucleobases and Nucleosides with Graphene. ChemPhysChem 2009, 10, 206-210.

(23) Huang, P.-J. J.; Liu, J., A Molecular Beacon Lighting up on Graphene Oxide. Anal. Chem. 2012, 84, 4192-4198.

(24) Qing, G.; Zhao, S.; Xiong, Y.; Lv, Z.; Jiang, F.; Liu, Y.; Chen, H.; Zhang, M.; Sun, T., Chiral Effect at Protein/Graphene Interface: A Bioinspired Perspective to Understand Amyloid Formation. J. Am. Chem. Soc. 2014, 136, 10736-10742.

(25) Gomez-Navarro, C.; Meyer, J. C.; Sundaram, R. S.; Chuvilin, A.; Kurasch, S.; Burghard, M.; Kern, K.; Kaiser, U., Atomic Structure of Reduced Graphene Oxide. Nano Lett. 2010, 10, 1144-1148.

(26) Erickson, K.; Erni, R.; Lee, Z.; Alem, N.; Gannett, W.; Zettl, A., Determination of the Local Chemical Structure of Graphene Oxide and Reduced Graphene Oxide. Adv. Mater. 2010, 22, 4467-4472.

(27) Wang, F.; Liu, B.; Ip, A. C. F.; Liu, J., Orthogonal Adsorption onto Nano-Graphene Oxide Using Different Intermolecular Forces for Multiplexed Delivery. Adv. Mater. 2013, 25, 4087-4092.

(28) Petrache, H. I.; Dodd, S. W.; Brown, M. F., Area per Lipid and Acyl Length Distributions in Fluid Phosphatidylcholines Determined by ${ }^{2} \mathrm{H}$ NMR Spectroscopy. Biophys. $J$. 2000, 79, 3172-3192.

(29) Li, Z. T.; Wang, Y. J.; Kozbial, A.; Shenoy, G.; Zhou, F.; McGinley, R.; Ireland, P.; Morganstein, B.; Kunkel, A.; Surwade, S. P.; Li, L.; Liu, H. T., Effect of Airborne Contaminants on the Wettability of Supported Graphene and Graphite. Nat. Mater. 2013, 12, 925-931.

(30) Li, S.; Stein, A. J.; Kruger, A.; Leblanc, R. M., Head Groups of Lipids Govern the Interaction and Orientation between Graphene Oxide and Lipids. J. Phys. Chem. C 2013, 117, 16150-16158.

(31) Fu, J.; Schlenoff, J. B., Driving Forces for Oppositely Charged Polyion Association in Aqueous Solutions: Enthalpic, Entropic, but Not Electrostatic. J. Am. Chem. Soc. 2016, 138, 980-990. 